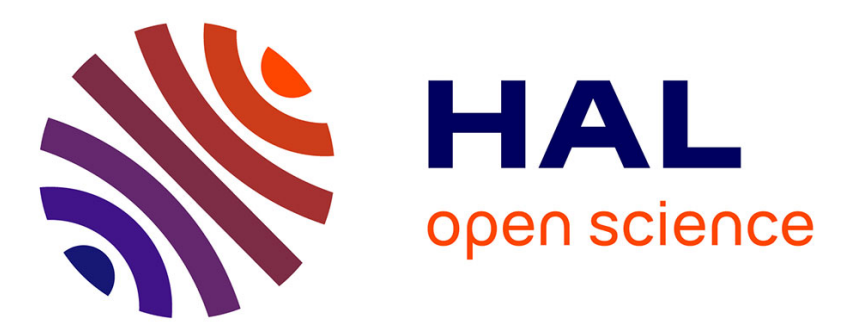

\title{
Degradation Modeling and Condition-Based Maintenance Decision-Making for Dynamic System in S-plane
}

Tran Minh Hao La, Khac Tuan Huynh, Antoine Grall, Yves Langeron

\section{- To cite this version:}

Tran Minh Hao La, Khac Tuan Huynh, Antoine Grall, Yves Langeron. Degradation Modeling and Condition-Based Maintenance Decision-Making for Dynamic System in S-plane. 29th European Safety and Reliability Conference (ESREL), Sep 2019, Hannover, Germany. 10.3850/978-981-11-2724-3 . hal-03249010

\section{HAL Id: hal-03249010 \\ https://hal-utt.archives-ouvertes.fr/hal-03249010}

Submitted on 3 Jun 2021

HAL is a multi-disciplinary open access archive for the deposit and dissemination of scientific research documents, whether they are published or not. The documents may come from teaching and research institutions in France or abroad, or from public or private research centers.
L'archive ouverte pluridisciplinaire HAL, est destinée au dépôt et à la diffusion de documents scientifiques de niveau recherche, publiés ou non, émanant des établissements d'enseignement et de recherche français ou étrangers, des laboratoires publics ou privés. 


\author{
T.M.H. LA, K.T. HUYNH, A. GRALL, Y. LANGERON \\ ICD, ROSAS, LM2S, Université de Technologie de Troyes, UMR 6281, Troyes, France \\ E-mail: tran_minh_hao.la@utt.fr,tuan.huynh@utt.fr,antoine.grall@utt.fr, yves.langeron@utt.fr
}

\begin{abstract}
Degradation modeling and maintenance decision-making based on degradation information are two majors issues in the field of reliability and maintenance research. This paper proposes an s-plane (complex plan) approach to deal with these two issues. A well-known mass-spring-damper dynamic system is considered as a case study. The loss of spring stiffness and of damper friction degrade the system performance over time. We show that the location of the system pole in the s-plane could be a good indicator to characterize the degradation of the whole system. Based on such an indicator, a maintenance policy is proposed to reduce as much as possible maintenance costs. We use the distance between the current and initial locations of the system pole to trigger maintenance on the system. Without any direct information on the components, we exploit the relative location of the system pole in the s-plane to decide which component (spring, damper or both) should be replaced. The performances of the proposed policy are pointed out via numerical comparisons with a more classical policy.
\end{abstract}

Keywords: condition-based maintenance, degradation indicator, dynamic system, s-plane, maintenance cost model.

\section{INTRODUCTION}

In the industrial context, maintenance operations play an important role to improve the durability, reliability, and maintainability of industrial systems. Various classifications of maintenance policies have been proposed in the literature (see e.g., Ding and Kamaruddin (2015)), and the most popular one distinguishes breakdown maintenance and time-based maintenance from condition-based maintenance (CBM). Over the last decade, CBM has been attracted by many researchers and practitioners because it responses very well to the current trend of Industry 4.0. In CBM, degradation modeling and maintenance decision-making based on degradation information are two major issues. If these issues have been extensively investigated at the component level (Alaswad and Xiang, 2017), their study at the system level is still scattered. Extending from the component level to the system level, one common approach is to take into account the dependencies (either stochastic, structural, or economic) among components (Keizer et al., 2017). Such an approach requires deep knowledge about the degradation of components and about their interactions, which is usually hard to achieve in practice. The present paper proposes an alternative approach (called s-plane) to deal with the degradation modeling and the maintenance decision-making of a dynamic system when information about the components degradation is latent.

The performances of a dynamic system can be reflected via the location of its dominant poles in s-plane (see e.g., Huynh et al. (2017)). Indeed, under the impacts of degradation phenomena, the system poles stochastically evolve in the s-plane far from their initial location (see e.g., Suyama and Sebe (2010)). This makes the system more and more different than its initial design, and hence unable to fulfill required missions. This means that the well-functioning of the system depends on the distance between the initial and current locations of the poles. If the distance exceeds a predefined value $L$, the system is considered as failed. As a result, the location of a system pole, as well as their associated distance can be seen as degradation indicators of the dynamic system.

Given degradation indicators of the system, we can determine a relevant date to trigger maintenance actions on the system. However, when direct information on the degradation of components is unavailable, what should we do on the components at a given date is a challenging question. In this paper, we seek the answer from the current location of the system pole. Indeed, having the same distance from its initial location, the location of the system pole in s-plane may be different depending on the degradation of components. In other words, some traces of the components degradation can be found in the location of the system pole. So, our objective is to exploit such information to decide whether or not to replace one or more components at an intervention time. Consequently, a maintenance policy with a two-level decision is proposed. A threshold is applied to the distance between the initial and current pole locations to decide whether or not to

Proceedings of the 29th European Safety and Reliability Conference.

Edited by Michael Beer and Enrico Zio

Copyright (c) 2019 European Safety and Reliability Association.

Published by Research Publishing, Singapore.

ISBN: 978-981-11-2724-3; doi:10.3850/978-981-11-2724-3_0501-cd 
trigger a maintenance action at the system-level. When a maintenance date has been determined, checking the deviation angle between the current and initial locations of the system pole allows us to choose the suitable component to be maintained.

In this paper, the proposed approach is applied to a mass-spring-damper dynamic system. The spring and the damper are two deteriorating components to be maintained. A cost model of the maintenance policy is analyzed, optimized and compared with the classical $(\Delta \tau, \xi)$ CBM policy proposed in Huynh et al. (2011). The comparison results allow us to assess the performance of the proposed approach and hence to make some conclusions on future work.

The remainder of this paper is organized as follow. Section 2 describes the mass-spring-damper dynamic system of interest. In Section 3, we determine some indicators characterizing the system degradation, and we define the system failure. A maintenance policy for the mass-spring-damper system is proposed in Section 4. Numerical experiments provided in Section 5 allow us to evaluate the performance of the maintenance policy. Finally, we conclude the paper in Section 6.

\section{SYSTEM DESCRIPTION}

We consider a well-known mass-spring-damper system (see e.g., Di Cairano et al. (2007)) whose spring and damper degrade over time due to the stiffness loss and the friction loss. The degradation evolution is assimilated as a homogeneous Gamma process.

\subsection{Mass-Spring-Damper System}

The mass-spring-damper system consists of a spring with stiffness $K$, a damper with friction $C$ and of a mobile mass $M$ (see Fig. 1).

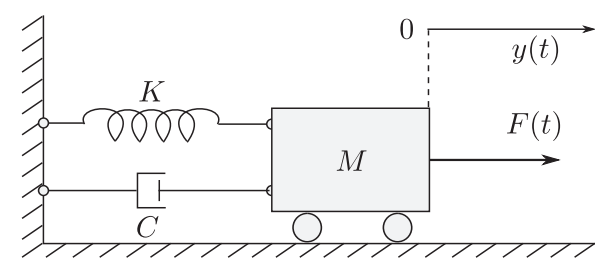

Fig. 1. Mass-spring-damper system

The system input is an external force $F(t)$ that triggers the mass in movement, and the system output is the shifting $y(t)$ of the mass around its balance position. The relation between the system input and output is described by using Newton's second law as follows

$$
M \frac{d^{2} y(t)}{d t^{2}}+C \frac{d y(t)}{d t}+K y(t)=F(t)
$$

Using the Laplace transform, we can deduce the transfer function

$$
H(p)=\frac{Y(p)}{F(p)}=\frac{1}{M p^{2}+C p+K} .
$$

The poles of the system are the roots of the characteristic equation (i.e., denominator of $H(p)$ )

$$
p_{1,2}=\frac{-C \pm \sqrt{C^{2}-4 M K}}{2 M} .
$$

When $C^{2}-4 M K<0$, the poles are complex conjugates whose real part and imaginary part are respectively

$$
P_{r}=-\frac{C}{2 M}, \quad P_{i}= \pm \frac{\sqrt{4 M K-C^{2}}}{2 M} .
$$

This is the common configuration of most dynamic system in practice. Since the two poles $p_{1}$ and $p_{2}$ have the same meaning, it is enough to consider the pole located above the real axis. We need to collect the location $\left(P_{r}, P_{i}\right)=$ $\left(-\frac{C}{2 M}, \frac{\sqrt{4 M K-C^{2}}}{2 M}\right)$ of the system pole for further study. To this end, we can trigger the system by a unit step input in a small time interval, and we record the associated output. Then, we apply the prediction error estimation method developed in Ljung (2002) to estimate the pole location.

\subsection{Degradation Sources}

For the considered mass-spring-damper system, the loss of spring stiffness and the loss of damping friction are the two primary degradation sources that decrease the system performance. In the literature, a degradation phenomenon can be modeled by either a deterministic approach or a stochastic approach (see e.g., Medjaher et al. (2014), Travé-Massuyès et al. (2015)). The deterministic choice does not integrate the intrinsic variability of each component. Meanwhile, the deterioration of material usually has a random nature in reality. Therefore, we will take advantage of stochastic modeling for the two above degradation sources.

A well-kown homogeneous Gamma process $\{X(t)\}_{t>0}$ parameterized by $\alpha$ and $\beta$ is used to model the loss evolution. According to Van Noortwijk (2009), $\{X(t)\}_{t>0}$ is a positive jump process, whose increments $X(t)-$ $X(s), 0 \leq s \leq t$, are independent and follow a Gamma probability density function (pdf) with shape parameter $\alpha(t-s)$ and scale parameter $\beta$

$$
f_{\alpha(t-s), \beta}(u)=\frac{\beta^{\alpha(t-s)} u^{\alpha(t-s)-1} e^{-\beta u}}{\Gamma(\alpha(t-s))},
$$


where $u \geq 0$ and $\Gamma(\cdot)$ is the complete Gamma function

$$
\Gamma(y)=\int_{0}^{\infty} u^{y-1} e^{-u} d u, \quad y>0 .
$$

The mean rate and variance rate of $\{X(t)\}_{t \geq 0}$ are $m=\alpha / \beta$ and $m=\alpha / \beta^{2}$. The couple of parameters $(\alpha, \beta)$ allows to model various evolution behaviors form almost-deterministic to verychaotic. With usage or age, the spring stiffness $K(t)$ and the damper friction $C(t)$ are decreasing over time so that we can express them as

$$
C(t)=C_{0}-X_{C}(t), K(t)=K_{0}-X_{K}(t),
$$

where $C_{0}$ and $K_{0}$ are the friction coefficient and the stiffness constant when the system is at the nominal mode. $X_{C}(t)$ and $X_{K}(t)$ are the accumulated loss at time $t \geq 0$ following homogeneous Gamma processes with parameters $\left(\alpha_{C}, \beta_{C}\right)$ and $\left(\alpha_{K}, \beta_{K}\right)$ respectively. These parameters can be estimated from the locations of the system pole (Huynh et al., 2017).

\section{DEGRADATION AND FAILURE}

\subsection{System Degradation Indicators}

This section aims at proposing suitable indicators to characterize the degradation state of the whole system. As shown in Bolton (1992), the system failure can directly be linked to the global resulting dynamic performances of the system. Thus, the location of a system pole in s-plane can properly gather all the system characteristics and notably its performances. To demonstrate more clearly this idea, we first define the evolution of $C(t)$ and $K(t)$ by the set of parameters: $C_{0}=$ $100, \alpha_{C}=0.54, \beta_{C}=0.75, K_{0}=100, \alpha_{K}=$ $0.54, \beta_{K}=0.75$. Next, we analyze how the evolution of $C(t)$ and $K(t)$ impact the movement of the system pole in s-plane. Fig. 2 and Fig. 3 represent respectively the results.

As it can be seen in Fig. 3, both the losses of the spring stiffness and of the damper friction in time (see Fig. 2) can make the system pole moves down and to the right in the s-plane towards the lower performance area (Maret, 1987). Moreover, the system pole tends to be far from its initial position for smaller values of $C(t)$ and $K(t)$. As such, the location of the system pole, as well as the distance between its current and initial locations could be good candidates for system degradation indicator. Using the location of the system pole as a degradation indicator, the evolution of the system degradation can be modeled by a bivariate stochastic process $\left\{P_{r}(t), P_{i}(t)\right\}_{t>0}$ where $P_{r}(t)$ and $P_{i}(t)$ represent respectively the real and imaginary parts of the considered dominant system pole. The random variables $P_{r}(t)$ and $P_{i}(t)$ can
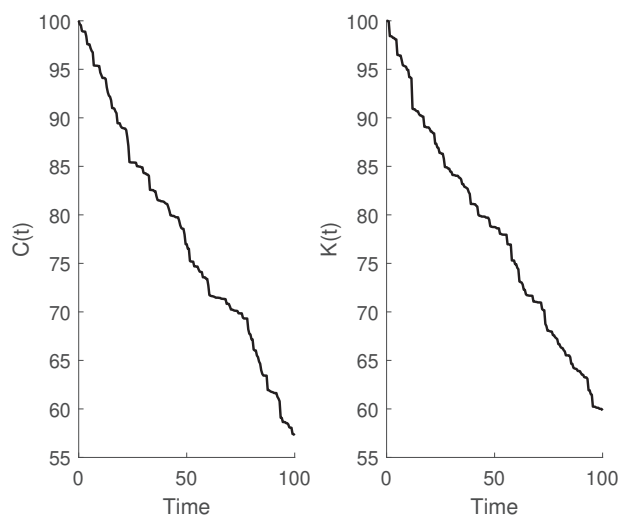

Fig. 2. Stochastic evolution of $C(t)$ and $K(t)$

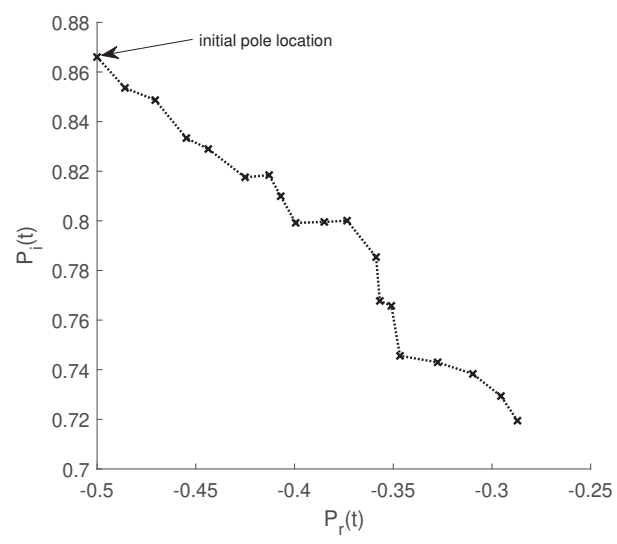

Fig. 3. Evolution of the system pole in s-plane

be expressed by

$$
P_{r}(t)=-\frac{C_{0}-X_{C}(t)}{2 M}
$$

$P_{i}(t)=\frac{\sqrt{4 M\left(K_{0}-X_{K}(t)\right)-\left(C_{0}-X_{C}(t)\right)^{2}}}{2 M}$

Moreover, since $P_{r}^{2}(t)+P_{i}^{2}(t)=\frac{K(t)}{M}$, we can derive from (8) and (9) that $P_{r}(t) \in\left[-\frac{C_{0}}{2 M} ; 0\right]$ and $P_{i}(t) \in\left[0 ; \sqrt{\frac{K_{0}}{M}}\right]$. The distance between its initial and current pole locations at time $t$ can be computed from $P_{r}(t)$ and $P_{i}(t)$ as

$$
D(t)=\sqrt{\left(P_{r}(t)-p_{r, 0}\right)^{2}+\left(P_{i}(t)-p_{i, 0}\right)^{2}}
$$

where $\left(p_{r, 0}, p_{i, 0}\right)$ denotes the initial location of the system pole. Assuming that $C(t)$ and $K(t)$ 
are independent, we look for the probability law of the two considered degradation indicators $\left(P_{r}(t), P_{i}(t)\right)$ and $D(t)$. Let $\left(P_{r}\left(t_{j}\right), P_{i}\left(t_{j}\right)\right)=$ $\left(p_{r, j}, p_{i, j}\right)$ be the location of the system pole at time $t_{j}$. By using the classical change of variables theorem, the joint pdf of the couple of variables $\left(P_{r}(t), P_{i}(t)\right)$ at time $t$ given $\left(p_{r, j}, p_{i, j}\right)$ can be computed as

$$
\begin{gathered}
f_{P_{r}(t), P_{i}(t) \mid P_{r}\left(t_{j}\right), P_{i}\left(t_{j}\right)}\left(p_{r}, p_{i} \mid p_{r, j}, p_{i, j}\right)= \\
4 M^{2} p_{i} f_{\alpha_{C}\left(t-t_{j}\right), \beta_{C}}\left(2 M\left(p_{r}-p_{r, j}\right)\right) \times \\
f_{\alpha_{K}\left(t-t_{j}\right), \beta_{K}}\left(M\left(p_{r, j}^{2}+p_{i, j}^{2}-p_{r}^{2}-p_{i}^{2}\right)\right),
\end{gathered}
$$

where $f_{\alpha_{C}\left(t-t_{j}\right), \beta_{C}}(\cdot)$ and $f_{\alpha_{K}\left(t-t_{j}\right), \beta_{K}}(\cdot)$ can be derived by Eq. (5). From Eq. (11), we can derive the conditional pdf of $D(t)$ given $\left(p_{r, j}, p_{i, j}\right)$ as

$$
\begin{aligned}
& f_{D_{t} \mid P_{r, t_{j}}, P_{i, t_{j}}}\left(d \mid p_{r, j}, p_{i, j}\right)= \\
& \int_{\mathcal{D}_{I}} \frac{d}{\sqrt{d^{2}-\left(p_{i}-p_{i, 0}\right)^{2}}} f_{P_{r, t}, P_{i, t} \mid P_{r, t_{j}}, P_{i, t_{j}}}\left(p_{r, 0}\right. \\
& \left.+\sqrt{d^{2}-\left(p_{i}-p_{i, 0}\right)^{2}}, p_{i} \mid p_{r, j}, p_{i, j} d p_{i}\right),
\end{aligned}
$$

where $\mathcal{D}_{I}=\left\{p_{i} \in \mathbb{R}^{+} \mid d^{2}-\left(p_{i}-p_{i, 0}\right)^{2}>0\right\}$.

\subsection{System Failure}

In many practical applications, a system failure occurs when there exists an important defect or a high deterioration that makes the system unable to fulfill its requirements. Following this idea, we consider the mass-spring-damper system as failed when its performance is too different from the initial design. Accordingly, we can define the failure threshold as a circle with radius of $L$ in the s-plane whose center is at the initial location of the system pole. When the system pole moves

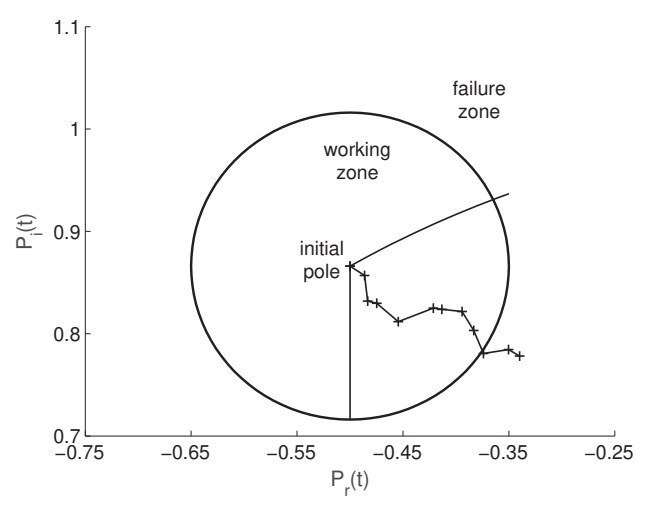

Fig. 4. Working-failure zone in s-plane out this circle, the system fails. Fig. 4 shows how the system pole evolves in the s-plane from the nominal state to the system failure. Fig. 5 depicts

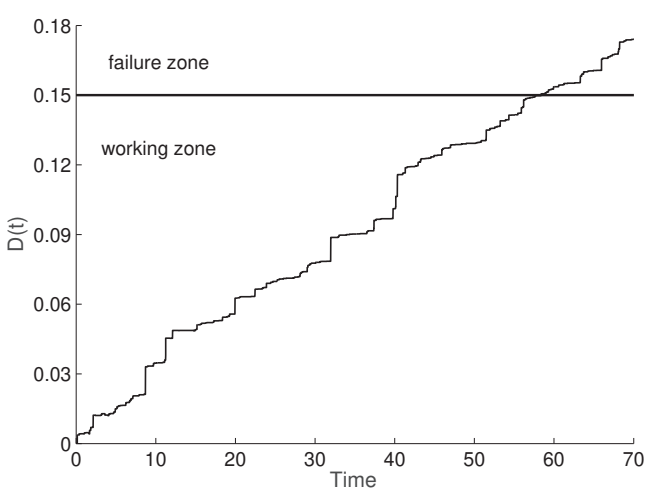

Fig. 5. Working-failure zone in time domain

the evolution in time of the associated distance. If the failure threshold associated with the system pole location is a circle in the s-plane, the one associated with the pole distance is a constant in time. Thanks to the monotony of the Gamma process and of the distance function (10), we can show easily that $D(t)$ is a monotone increasing process in time (see Fig. 5).

\section{MAINTENANCE POLICIES}

We propose in this section a new maintenance policy for the considered mass-spring-damper system based on both the distance and the location of the system pole. Its long-run maintenance cost rate is evaluated using the Monte Carlo simulation approach.

\subsection{Assumptions and Maintenance Costs}

We assume that system inspection and replacement as new of the components (either the spring, the damper or both) are the two available maintenance actions for the system. The inspection, the replacement of the spring and of the damper incur $C_{i}, C_{K}$, and $C_{C}$ cost unit respectively. The inspection can return the current location of the system pole, but cannot reveal any direct information about the degradation of components. Although only the perfect replacement is applied to the components, there exist, at the system level, both the perfect and imperfect maintenance actions. If both the spring and damper are replaced at the same maintenance date, the system is asgood-as-new. If one of them is replaced, the system is imperfectly repaired. We also distinguish between preventive maintenance and corrective 
maintenance. The former is done when the system is still working, while the latter is performed on a failed system. Compared to the preventive maintenance, the corrective maintenance is likely more complex and may induce damage to the environment and other systems. So, in addition to the cost of spring and damper replacement (i.e., $C_{K}+C_{C}$ ), a penalized cost $C_{p}$ is added for each corrective maintenance. Moreover, since the system is unavailable after failure, an additional downtime cost is taken from the failure time until the next intervention time at a cost rate $C_{d}$.

\section{2. $(\Delta \tau, \xi)$ Policy}

When the information about the degradation of components is unavailable, a classical way to build a maintenance policy is to consider the multi-component system as a single unit (Grall et al., 2002). Therefore, the $(\Delta \tau, \xi)$ policy proposed in Huynh et al. (2011) can be applied. The system is regularly inspected with period $\Delta \tau$. At an inspection time $\tau_{k}=k \Delta \tau$, given the location $\left(P_{r}\left(\tau_{k}\right), P_{i}\left(\tau_{k}\right)\right)=\left(p_{r, k}, p_{i, k}\right)$ of system pole, we evaluate the associated distance $D\left(\tau_{k}\right)$ from its initial location, and adopt the following decision rule.

(1) If $D\left(\tau_{k}\right) \geq L$, the system has been failed, so we have to perform a corrective maintenance. After the replacement of both the spring and damper at $\tau_{k}$, the system is as good as new.

(2) If $\xi \leq D\left(\tau_{k}\right)<L$, the system is still working but highly deteriorated, we should carry out a preventive maintenance. After the replacement of both the spring and damper at $\tau_{k}$, the system is as good as new.

(3) If $0<D\left(\tau_{k}\right)<\xi$, the system is still healthy, additional actions at $\tau_{k}$ is useless. Consequently, the system state is unchanged.

In all cases, the next inspection time is $\tau_{k+1}=$ $\tau_{k}+\Delta \tau$, at which the same decision structure is repeated. For this policy, the inspection period $\Delta \tau$ and the preventive replacement threshold $\xi$ are the two decision parameters to be optimized. Fig. 6 shows sample evolution paths of $C(t), K(t)$ and $D(t)$ in time when the $(\Delta \tau, \xi)$ policy is used.

\section{3. $\left(\Delta \tau, \xi, \theta_{C}, \theta_{K}\right)$ Policy}

The above $(\Delta \tau, \xi)$ policy is simple, but not economically performant, because both the spring and damper are replaced whenever a maintenance is needed. It seems more effective if we can decide which one between them should be replaced at a preventive maintenance date. Such decisions require information about the degradation of the spring and damper. However, when these informations cannot be gathered by additional inspection actions due to e.g., technical or economic reasons, we should extract them from the location

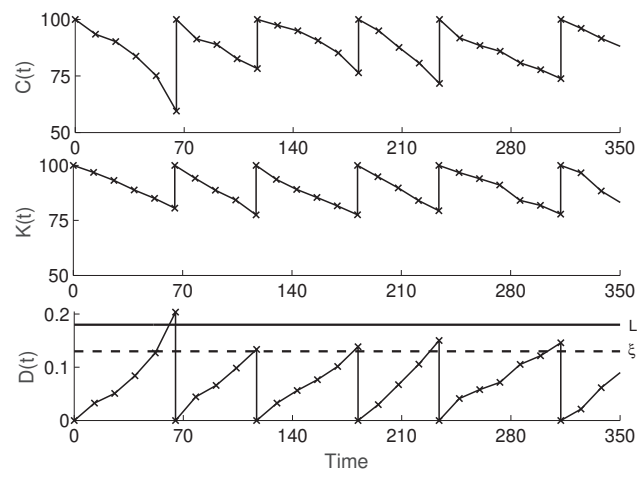

Fig. 6. System behavior under the $(\Delta \tau, \xi)$ policy

of the system pole in s-plane. Indeed, looking at Eq. (8) and Eq. (9), we find that the loss of spring stiffness affects only the imaginary part of the system pole. Accordingly, if the system pole locates in the zone I (see Fig. 7), the spring should be replaced, because only the imaginary part of the system pole is critical in terms of system degradation. Similarly, it is better to replace only

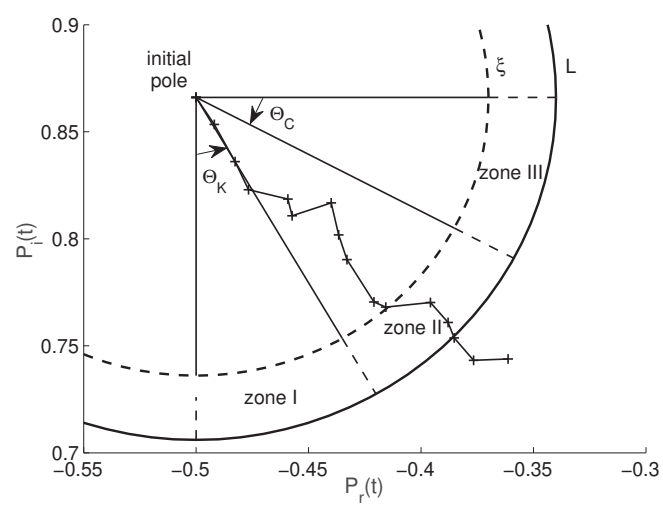

Fig. 7. Preventive maintenance division at component-level

the damper if the pole locates in the zone III, and to replace both the spring and damper if it is in zone II. As a consequence, we can define two thresholds called $\theta_{K}$ and $\theta_{C}$ associated with the angles defining zone I and III (see Fig. 7) to choose the suitable components to be replaced at a preventive maintenance date.

Extending the $(\Delta \tau, \xi)$ policy, we state a new maintenance policy for the mass-spring-damper system as follows. The system is also regularly inspected with period $\Delta \tau$. At an inspection time $\tau_{k}=k \Delta \tau$, we evaluate the distance $D\left(\tau_{k}\right)$, and adopt the following decision rule. 
(1) If $D\left(\tau_{k}\right) \geq L$, the system has been failed, and a corrective maintenance have to be done. After the replacement of both the spring and damper at $\tau_{k}$, the system is as good as new.

(2) If $\xi \leq D\left(\tau_{k}\right)<L$, the system is still working but highly deteriorated, therefore a preventive maintenance should be carried out. Depending on the relative relation between the location $\left(p_{r, k}, p_{i, k}\right)$ and the angles $\theta_{K}$ and $\theta_{C}$, we decide the components to be replaced as follows.

- If $\left|\frac{P_{r}(t)-p_{r, 0}}{P_{i}(t)-p_{i, 0}}\right| \leq \tan \left(\theta_{K}\right)$, only the spring is replaced.

- If $\left|\frac{P_{i}(t)-p_{i, 0}}{P_{r}(t)-p_{r, 0}}\right| \leq \tan \left(\theta_{C}\right)$, only the damper is replaced.

- Otherwise, both the spring and damper shoud be replaced.

After the replacement at $\tau_{k}$, only in the last case the system is as good as new, in the other case, it is better than old but worse than new.

(3) If $0<D\left(\tau_{k}\right)<\xi$, the is still healthy, and no additional action at $\tau_{k}$ is required. As a results, the system state is unchanged.

In all cases, the next inspection time is $\tau_{k+1}=$ $\tau_{k}+\Delta \tau$, at which the same decision structure is repeated. $\Delta \tau, \xi, \theta_{C}$ and $\theta_{K}$ are the four decision parameters to be optimized of this new policy. We shall call it $\left(\Delta \tau, \xi, \theta_{C}, \theta_{K}\right)$ policy to highlight the importance of these parameters. Sample evolution paths of $C(t), K(t)$ and $D(t)$ under the $\left(\Delta \tau, \xi, \theta_{C}, \theta_{K}\right)$ policy are shown in Fig. 8. Obvi-
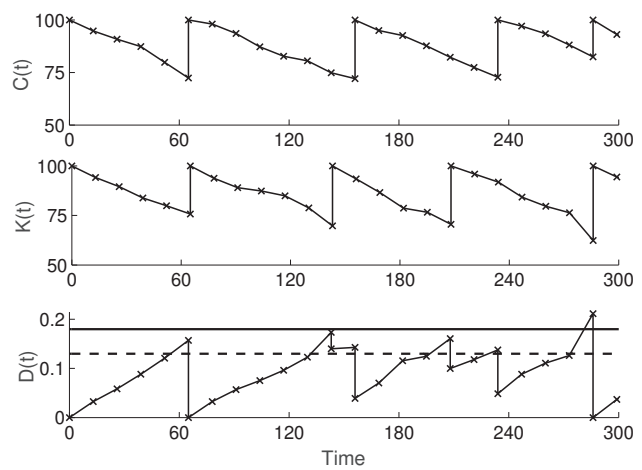

Fig. 8. System behavior under the $\left(\Delta \tau, \xi, \theta_{C}, \theta_{K}\right)$ policy

ously, the $\left(\Delta \tau, \xi, \theta_{C}, \theta_{K}\right)$ policy is more flexible, and hence more profitable than the $(\Delta \tau, \xi)$ policy. Moreover, there is no risk in using the new one because it returns to the more classical policy in the worse case (i.e., $\theta_{C}=\theta_{K}=0$ ).

\subsection{Cost Criterion and Optimization}

A classical criterion to assess the performance of a maintenance policy is the long-run maintenance cost rate defined by

$$
C_{\infty}=\lim _{t \rightarrow \infty} \frac{C(t)}{t},
$$

where $C(t)$ denotes the accumulated maintenance cost up to time $t$. For the $\left(\Delta \tau, \xi, \theta_{C}, \theta_{K}\right)$ policy, the cost rate $C_{\infty}$ can be analytically evaluated in the same way as Huynh et al. (2015). Because of the paper missing, we omit the analytical formulation, and apply just the Monte Carlo simulation to obtain $C_{\infty}$.

For the purpose of comparison with the $(\Delta \tau, \xi)$ policy, a two-step optimization procedure has been applied to the $\left(\Delta \tau, \xi, \theta_{C}, \theta_{K}\right)$ policy (see Fig. 9). Firstly, we minimize the cost rate

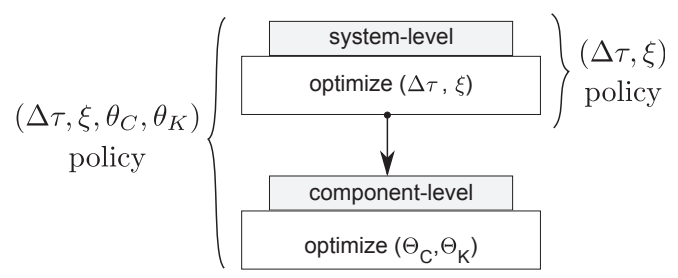

Fig. 9. Optimization for the $\left(\Delta \tau, \xi, \theta_{C}, \theta_{K}\right)$ policy

$C_{\infty}^{(\Delta \tau, \xi)}$ of the $(\Delta \tau, \xi)$ policy to obtain the optimal values of the inspection period and the preventive maintenance threshold $\left(\Delta \tau_{o p t}^{o}, \xi_{o p t}^{o}\right)$. Any derivative free algorithms for black-box optimization (Audet and Hare, 2017) can be used here. Next, for $\left(\Delta \tau, \xi, \theta_{C}, \theta_{K}\right)$ policy, we set $\Delta \tau=\Delta \tau_{\text {opt }}^{o}$ and $\xi=\xi_{\text {opt }}^{o}$, and we search the optimal couple of angles $\left(\theta_{C, \text { opt }}, \theta_{K, \text { opt }}\right)$ that minimizes the cost rate $C_{\infty}^{\left(\Delta \tau_{o p t}^{o}, \xi_{o p t}^{o}, \theta_{C}, \theta_{K}\right)}$. Of course, this procedure penalizes the optimal cost rate of the $\left(\Delta \tau, \xi, \theta_{C}, \theta_{K}\right)$ policy, because their decision parameters are not jointly optimized. However, if we can find $\left(\theta_{C, o p t}, \theta_{K, o p t}\right)$ verifying $C_{\infty}^{\left(\Delta \tau_{o p t}^{o}, \xi_{o p t}^{o}, \theta_{C, o p t}, \theta_{K, o p t}\right)}<C_{\infty}^{\left(\Delta \tau_{o p t}^{o}, \xi_{o p t}^{o}\right)}$, it is enough to conclude the higher performance of the $\left(\Delta \tau, \xi, \theta_{C}, \theta_{K}\right)$ policy.

\section{NUMERICAL EXPERIMENTALS}

This section aims at illustrating and analyzing the advantage of the $\left(\Delta \tau, \xi, \theta_{C}, \theta_{K}\right)$ policy through numerical experimentals. To this end, we consider a mass-spring-damper system defined by the set of parameters $C_{0}=100, \alpha_{C}=0.54, \beta_{C}=0.75$, $K_{0}=100, \alpha_{K}=0.54, \beta_{K}=0.75$ and $M=$ 100. This is the same system in Section 3 so that sample paths of $C(t), K(t)$, and of $\left(P_{r}(t), P_{i}(t)\right)$ 
can be found in Fig. 2 and Fig. 3. The maintance costs are chosen by $C_{i}=2, C_{C}=25, C_{K}=25$, $C_{p}=50$ and $C_{d}=100$.

Applying the optimization procedure in Section 4.4, we seek firstly the optimal configuration of the $(\Delta \tau, \xi)$ policy. As shown in Fig. 10, such a configuration exists at $\left(\Delta \tau_{\text {opt }}^{o}, \xi_{\text {opt }}^{o}\right)=(13,0.13)$ with $C_{\infty}^{\left(\Delta \tau_{o p t}^{o}, \xi_{o p t}^{o}\right)}=1.0371$.

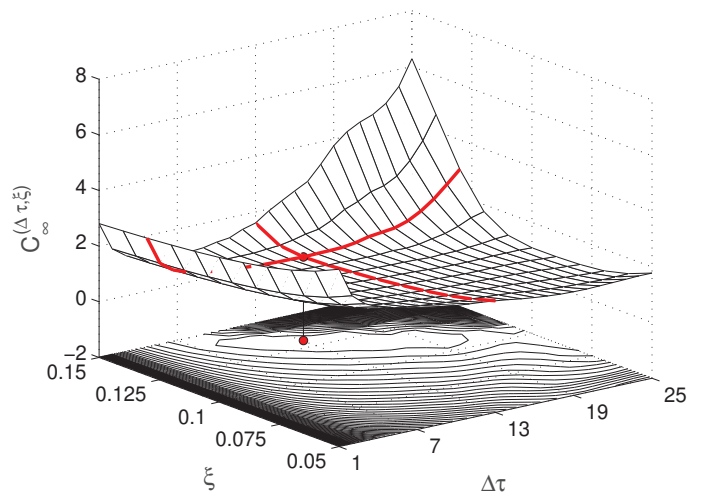

Fig. 10. Shape of the cost rate $C_{\infty}^{\left(\Delta \tau_{o p t}^{o}, \xi_{o p t}^{o}\right)}$

Fixing $(\Delta \tau, \xi)=\left(\Delta \tau_{\text {opt }}^{o}, \xi_{\text {opt }}^{o}\right)$, we obtain in Fig. 11 the long-run cost rate of the $\left(\Delta \tau, \xi, \theta_{C}, \theta_{K}\right)$ policy when $\theta_{C}$ and $\theta_{K}$ vary. The

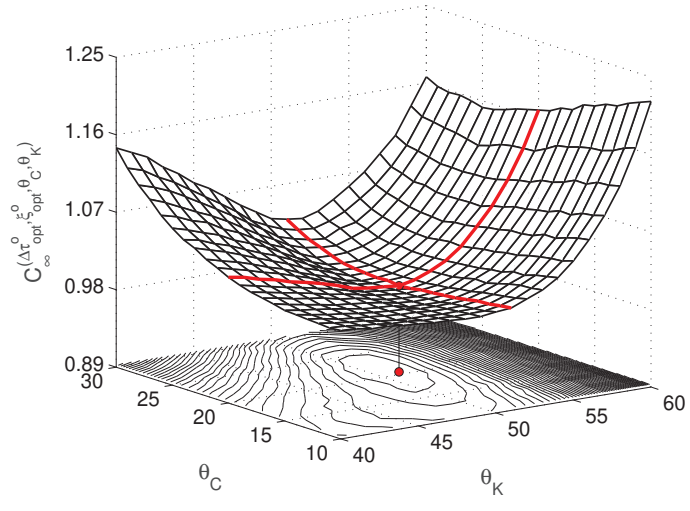

Fig. 11. Shape of the cost rate $C_{\infty}^{\left(\Delta \tau_{o p t}^{o}, \xi_{o p t}^{o}, \theta_{C}, \theta_{K}\right)}$

convex form of $C_{\infty}^{\left(\Delta \tau_{o p t}^{o}, \xi_{o p t}^{o}, \theta_{C}, \theta_{K}\right)}$ imply that optimal value of the couple $\left(\theta_{C}, \theta_{K}\right)$ exists. Indeed, we obtain $\left(\theta_{C, \text { opt }}, \theta_{K, \text { opt }}\right)=\left(21^{\circ}, 50^{\circ}\right)$ at which $C_{\infty}^{\left(\Delta \tau_{o p t}^{o}, \xi_{o p t}^{o}, \theta_{C, o p t}, \theta_{K, o p t}\right)}=0.9802$. This result confirms that the $\left(\Delta \tau, \xi, \theta_{C}, \theta_{K}\right)$ policy is more profitable than the $(\Delta \tau, \xi)$ policy when $C_{C}=25$ and $C_{K}=25$.

We would like to kown if the above result remains unchanged when $C_{C}$ and/or $C_{K}$ are modified. To avoid repeating the optimization of the $(\Delta \tau, \xi)$ policy many times, we always fix the sum $C_{C}+C_{K}=50$ (so that $\left(\Delta \tau_{\text {opt }}^{o}, \xi_{\text {opt }}^{o}\right)=$ $(13,0.13))$, vary $C_{K}$ from 5 to 45 with step 5 , jointly optimize $\left(\theta_{C}, \theta_{K}\right)$, and then observe the associated $C_{\infty}^{\left(\Delta \tau_{o p t}^{o}, \xi_{o p t}^{o}, \theta_{C, \text { opt }}, \theta_{K, \text { opt }}\right)}$. The results of this study are reported in Table 1 . We find

Table 1. Sensitivity study to the variation of $C_{K}$

\begin{tabular}{rlll}
\hline$C_{K}$ & $\theta_{C, \text { opt }}$ & $\theta_{K, \text { opt }}$ & $C_{\infty}$ \\
\hline 5 & 16 & 52 & 0.9598 \\
10 & 19 & 52 & 0.9718 \\
15 & 20 & 52 & 0.9796 \\
20 & 20 & 52 & 0.9862 \\
25 & 21 & 50 & 0.9891 \\
30 & 21 & 50 & 0.9983 \\
35 & 21 & 50 & 1.0034 \\
40 & 21 & 49 & 1.0084 \\
45 & 20 & 41 & 1.0116 \\
\hline
\end{tabular}

that when $C_{K}$ increases (i.e., $C_{C}$ decreases), the $\left(\Delta \tau, \xi, \theta_{C}, \theta_{K}\right)$ policy adjusts the angles $\theta_{K, o p t}$ smaller and $\theta_{K \text {,opt }}$ larger respectively. This adjustment allows to avoid the high cost due to the spring replacements while benefit the low replacement cost of the damper. Thank to this flexibility, the cost rate $C_{\infty}^{\left(\Delta \tau_{o p t}^{o}, \xi_{o p t}^{o}, \theta_{C, o p t}, \theta_{K, o p t}\right)}$ is slightly modified even for sudden changes increment in replacement cots of components. More importantly, the cost rate $C_{\infty}^{\left(\Delta \tau_{o p t}^{o}, \xi_{o p t}^{o}, \theta_{C, o p t}, \theta_{K, o p t}\right)}$ is still smaller than $C_{\infty}^{\left(\Delta \tau_{o p t}^{o}, \xi_{o p t}^{o}\right)}$ for whatever value of $C_{K}$. So, the economic performance of the $\left(\Delta \tau, \xi, \theta_{C}, \theta_{K}\right)$ policy is confirmed.

\section{CONCLUSIONS}

We have proposed in this paper a new approach (called s-plane) for degradation modeling and maintenance decision-making of a dynamic system with multiple components. We have shown that the location of the system pole in s-plane and the associated distance from its initial location are good indicators to characterize the degradation of the whole system. Furthermore, we may find useful information about the degradation of components through the pole location. We have exploited such information to build a twolevel maintenance policy (i.e., system level and component level) allowing to determining suitable components to be replaced without any additional inspection of their degradation. The policy has 
been proved more profitable than the maintenance policies applied to the system-level only.

\section{References}

Alaswad, S. and Y. Xiang (2017). A review on condition-based maintenance optimization models for stochastically deteriorating system. Reliability Engineering \& System Safety 157, 54-63.

Audet, C. and W. Hare (2017). Derivative-free and blackbox optimization. Springer.

Bolton, W. (1992). Control Engineering. Longman Scientific \& Technical.

Di Cairano, S., A. Bemporad, I. Kolmanovsky, and D. Hrovat (2007). Model predictive control of magnetic automotive actuators. In American Control Conference, 2007. ACC'07, pp. 50825087. IEEE.

Ding, S.-H. and S. Kamaruddin (2015). Maintenance policy optimizationliterature review and directions. The International Journal of Advanced Manufacturing Technology 76(5-8), 1263-1283.

Grall, A., L. Dieulle, C. Bérenguer, and M. Roussignol (2002). Continuous-time predictivemaintenance scheduling for a deteriorating system. IEEE Transactions on Reliability 51(2), 141-150.

Huynh, K. T., A. Barros, and C. Bérenguer (2015). Multi-level decision-making for the predictive maintenance of $k$-out-of- $n$ : $\mathrm{F}$ deteriorating systems. IEEE transactions on Reliability 64(1), 94-117.

Huynh, K. T., A. Barros, C. Bérenguer, and I. T. Castro (2011). A periodic inspection and replacement policy for systems subject to competing failure modes due to degradation and traumatic events. Reliability Engineering \& System Safety 96(4), 497-508.

Huynh, K. T., Y. Langeron, and A. Grall (2017). Degradation modeling and rul estimation of deteriorating systems in s-plane. IFACPapers OnLine 50(1), 12249-12254.

Keizer, M. C. O., S. D. P. Flapper, and R. H. Teunter (2017). Condition-based maintenance policies for systems with multiple dependent components: A review. European Journal of Operational Research 261(2), 405-420.

Ljung, L. (2002). Prediction error estimation methods. Circuits, Systems and Signal Processing 21(1), 11-21.

Maret, L. (1987). Régulation automatique. Presses polytechniques romandes.

Medjaher, K., H. Skima, and N. Zerhouni (2014). Condition assessment and fault prognostics of microelectromechanical systems. Microelectronics Reliability 54(1), 143-151.

Suyama, K. and N. Sebe (2010). Probabilistic safety management of control laws against deviations from normal operating-range. In Con- trol and Fault-Tolerant Systems (SysTol), 2010 Conference on, pp. 442-449. IEEE.

Travé-Massuyès, L., R. Pons, P. Ribot, Y. Pencolé, and C. Jauberthie (2015). Condition-based monitoring and prognosis in an error-bounded framework. In DX@ Safeprocess, pp. 83-90.

Van Noortwijk, J. (2009). A survey of the application of gamma processes in maintenance. Reliability Engineering \& System Safety 94(1), 2-21. 